\title{
The History and Advanced Technology of Plant Factories
}

Plant factories can be defined as horticulture greenhouses or as automated system facilities that produce vegetables and seedlings year round by controlling environmental conditions such as, light, temperature, humidity, $\mathrm{CO}_{2}$, and nutrient solutions. Recently, within plant factories, advanced technologies have been used to automatically adjust and control the growth environment. The main benefits of modern plant factory technologies are safety, security, and stable food supplies. They can address the ever-increasing problems of agricultural employee reduction, poor harvests due to abnormal weather resulting from global warming, and food shortages due to over population. As a result, advancements in agricultural business can be expected.

Plant factories can be broadly categorized as either artificial lighting based fully enclosed systems and natural sunlight based systems. The main cultivation method used in enclosed plant factories is hydroponics, while natural sunlight systems can use both soil and hydroponics technologies. Sunlight based plant factories can either use exclusively natural sunlight, or a combination of natural sunlight and artificial light.

In a closed-system plant factory, operating costs are high. While this method is not suitable to grow large fruits and vegetables, leafy vegetables are applicable. Small spaces, inside buildings or former industrial factories, are sufficient locations for plant growth systems. If the environmental control is optimal, the plants' nutritional value can be increased. On the other hand, sunlight based plant factories have a lower running cost compared to closed systems. They are more suitable for growing larger fruits and vegetables, but environmental control is difficult because of unpredictable climate changes.

The history of the plant factory and typical transitions are as follow:

In 1949, the Earhart Plant Research Laboratory at the California Institute of Technology in Pasadena developed the first greenhouse with control of lighting, temperature, humidity, $\mathrm{CO}_{2}$, wind, rain, and mist. This type of greenhouse used for research is called a phytotron.

During the 1950's in Japan, phytotrons were installed in universities, biological, and agricultural research institutes. In 1952, the environmental regulation greenhouse at the National Institute of Genetics became the country's first phytotron. In 1957, the agricultural faculty at the University of Tokyo installed a biological environmental control facility (biotron) that was able to control temperature, humidity, and artificial lighting. It was not only a phytotoron, but also an animal and insect environmental control laboratory for biological research purposes.

During the same time, in the 1950's and 60's, the BIOS-3 CELSS (Controlled Ecological Life Support Systems) began as a space development program in other countries. In 1967, the University of Wisconsin also established a facility named Biotron.

During the early 1970's in Japan, Hitachi Ltd. (currently the honorary member of this association (Japanese Society of Agricultural, Biological and Environmental Engineers and Scientists), Takatsuji Masaki) was the first in the world to begin test runs with plant factory technologies.

In the 1980's in America, large scale automated plant factories using natural sunlight became widespread. At the same time, in the Netherlands, plant production factories using artificial light as a supplement to grow flowers, ornamental plants, and seedlings also became prominent. In Japan, SPA (Speaking Plant Approach) biometric cultivation technologies were proposed by Hashimoto Yasushi, an Ehime University professor at this time.

In 1990, a plant factory inside the International Space Station was proposed, and research on the relationship between zero-gravity and plant growth began with the Salad Machine developed by NASA.

During this time in Japan, the aim was to improve production efficiency. Because of this focus, new techniques such as, fluorescent lighting based multiple-shelve cultivation systems, effective use of area for denser plant layouts, and cultivation panels floating on a flood bed have been developed. Robots were also introduced into plant factories where tests on seeding, harvesting and packing began and continue to the present day.

In 2008, a Japanese national policy known as the "Economic Growth Strategy for Widespread Plant Factory Use" was launched to promote the spread of completely controlled environmental and solar based plant factory businesses. In response to the third boom of 2009, the Mitsubishi Research Institute Inc. survey from March, 2012 showed that various plant factories were established and operations had already begun. 106 factories used exclusively artificial light, 21 used a combination of artificial and natural light, and 84 used exclusively natural sunlight. Since that time, rapid development on elements of automation technology management from cultivation to harvest has been ongoing, starting with environmental control.

Until now, it was presumed that only the machines were moving inside of plant factories. However, plant moving systems have also been recently analyzed for soil cultures. For example, the multi-staged lettuce cultivation system robot at Osaka Prefecture University, or the strawberry harvesting robot at the National Agricultural and Food Research Organization. 
The Netherlands greenhouse systems, currently spread around the world, are suitable for automation due to standardized facilities and cultivation methods. The spread of newly developed robots and machines is expected because the equipment was intended for general purpose use (for example, the tomato cultivation greenhouse at Ehime University).

There are various forms of completely controlled plant factories, depending on cultivar type, size, etc. They are commonly built from the ground up and have a high degree of flexibility because the systems use robots and automated machinery. Due to these factors, there are many possibilities for completely controlled plant factories into the future.

In recent years, the sixth sector industrialization has been drawing the attention of building agricultural production foundations to begin using plant factories in natural disaster-stricken areas. Therefore, innovations for next-generation hightech plant factory computerization and system controls are important.

The major challenges for plant factory advancement are listed below:

1) Establishing an academic setting to nurture the goal of environmentally controlled farming

2) Meeting the demand for environmental control using biometrics and SPA principles

3) Improving the scientific approach to plant cultivation process systems

4) Training researchers and engineers responsible for next-generation plant factories

Japan's automated artificial and natural sunlight type plant factories, and elemental technologies related to robotization, are state-of-the-art. In this special issue, original papers on the latest plant factory case studies are introduced in the first half, while the second half pertains to technical reports from domestic companies on advanced technology installations.

Junji Hirama

Fully Automated Plant Factory Committee 